\title{
Case Report: Metachronous Quadruple Cancers Including Breast Cancer and Triple Genital Cancer
}

This article was published in the following Dove Press journal:

International Journal of General Medicine

\begin{abstract}
Jing $\mathrm{Bai}^{\mathrm{l}, *}$
Zhaoguang $\mathrm{Xie}^{1, *}$

Li Sun ${ }^{2}$

'Department of Maternity, Jinan Maternity and Child Care Hospital Affiliated to Shandong First Medical University, Jinan 25000I, People's Republic of China; ${ }^{2}$ Department of Gynecological Oncology, Qingdao Central Hospital, The Second Affiliated Hospital of Medical College of Qingdao University, Qingdao 266042, People's Republic of China
\end{abstract}

*These authors contributed equally to this work
Correspondence: Li Sun

Tel +86-18953109686

Email sunli766@I63.com

\begin{abstract}
Most multiple primary cancers(MPCs) are double primaries, while quadruple cancer is rare, especially breast cancer and triple genital cancer. Our work is the first report about ametachronous quadruple cancer in the reproductive system, including breast, ovary, fallopian tube and cervix. A54-year-old woman, who underwent modified radical mastectomy and right breast tumor resection 3 years ago, presented with abnormal vaginal discharge for 2 months, and she was subsequently diagnosed with stage IIA2 cervical squamous cell carcinoma. She then underwent radiotherapy and chemotherapy, as well as radical hysterectomy, bilateral salpingo-oophorectomy and bilateral pelvic lymphadenectomy. Postoperative pathological findings revealed that the patient had cervical, ovarian and tubal triple cancers with different histopathology for all lesions, also different from breast cancer, implying that these tumors had separate etiology and were not metastatic tumors. Our work might provide aseries of diagnostic, etiological and therapeutic strategies for MPC patients. Keywords: quadruple cancer, multiple primary carcinoma, metachronous cancer, genital cancer
\end{abstract}

\section{Introduction}

Multiple primary cancers (MPCs), also known as multiple primaries, refer to the occurrence of more than two primary, synchronous or metachronous cancers in single or multiple organs in the same individual. ${ }^{1}$ In previous published review, the incidence of multiple primaries was reported between $2.4 \%$ and $8 \%$ depending on the cancer population, while in patients with breast cancer the incidence of multiple primaries was approximately $4.1 \%{ }^{1}$ However, most are double primary cancers, and quadruple cancer in asingle patient was rare, about $<0.1 \%{ }^{2}$ In the present work, we describe acase of metachronous MPC, over aperiod of more than 6 months. We herein report the female patient who presented with quadruple cancer of the reproductive system, which involved breast, cervix, ovary and oviduct.

\section{Case Report}

On May31, 2015, a51-year-old female patient was admitted to the Affiliated Hospital of Jining Medical College with amass on the right breast with the size of about an egg yolk, which was present for more than 20 days with no other discomfort. The patient had been suffering from lumbar disc herniation and hepatitis Bvirus for more than 10 years, and no standardized treatment had been given. Family history showed that her father died of liver cancer and her aunt was asurvivor of ovarian cancer. After admission, the patient underwent relevant 
auxiliary examinations. Color ultrasonography revealed asolid lesion in the right breast, BI-RADS grade IV. Computerized tomography (CT) confirmed the presence of amass in the right breast. Subsequently, she received modified radical mastectomy at the Affiliated Hospital of Jining Medical College, and her tumor was classified as T1N0M0 and stage Iaccording to the surgical findings. Histopathology of the tumor showed invasive ductal carcinoma. Immunohistochemical examination showed that tumor cells were negative for estrogen as well as progesterone receptors. The patient received eight cycles of combination chemotherapy consisting of $160 \mathrm{mg}$ docetaxel, $70 \mathrm{mg}$ D1D2 epirubicin and $900 \mathrm{mg}$ cyclophosphamide (TEC), and each cycle lasted for 3 weeks.

After 3 years, on May5, 2018, the patient presented herself at alocal county hospital with abnormal vaginal discharge for 2 months, with no cause for posterior vaginal bleeding. TCT (ThinPrep cytologic test) detected squamous cell carcinoma and positivity for HPV-16. She had a3-year history of diabetes with poor glycemic control due to self-administration of "metformin $0.5 \mathrm{mg}$ po bid and repaglinide $1 \mathrm{mg}$ po qd". Gynecological examination in our hospital revealed no abnormalities in the vulva or urethra; good vaginal dilatation, vaginal anterior hernia, right iliac crest; cervix about $5 \mathrm{~cm} \times 4 \mathrm{~cm}$, cauliflower-like, crisp, blood-positive; normal-sized uterus, good activity; soft side of the bilateral side, good elasticity; rectal mucosa of the anus was smooth with no obvious abnormality, and the blood test was negative. The CT scan suggested cervical cancer, and acervical biopsy confirmed papillary squamous cell carcinoma with poor differentiation (Figure 1). The carcinoma was classified as T2aNOM0 and stage IIa2 (according to the criteria of the International Federation of Gynecology and Obstetrics, FIGO). The patient received intravenous adjuvant chemotherapy with 200mg paclitaxel liposome and $120 \mathrm{mg}$ nedaplatin for one cycle, as well as one single intracavitary brachytherapy consisting of $500 \mathrm{cGy}$ at reference point A.Following radiotherapy and chemotherapy, the patient underwent radical hysterectomy, bilateral salpingo-oophorectomy, and bilateral pelvic lymphadenectomy. No significant surgical or medical complications occurred during these above-mentioned procedures. All surgical specimens were examined by agynecologic pathologist at our institution. Histopathologic findings indicated cervical squamous cell carcinoma with $>2 / 3$ myometrial invasion, left ovarian serous borderline tumors with intraductal carcinoma and right fallopian tube adenocarcinoma (Figure 2A-C). No invasion of the lymphovascular space was identified. Based on pathologic findings, simultaneous radiotherapy and chemotherapy were given. The parameters for invitro intensity-modulated radiotherapy were set to $6 \mathrm{MV} \mathrm{X}$-rays at adose of $1080 \mathrm{~Gy} / 6$ Ffor the pelvic lymph node drainage area; and adose of $3960 \mathrm{~Gy} / 22$ Fwas given for the abdominal pelvic lymph node drainage area and the vaginal stump target area. Treatments were set to $(1,1)$ point at 700 Gy for three treatments. Additionally, intravenous chemotherapy was implemented with $210 \mathrm{mg}$ liposomal paclitaxel and $150 \mathrm{mg}$ oxaliplatin for one cycle. Because of the degree of bone marrow suppression associated with

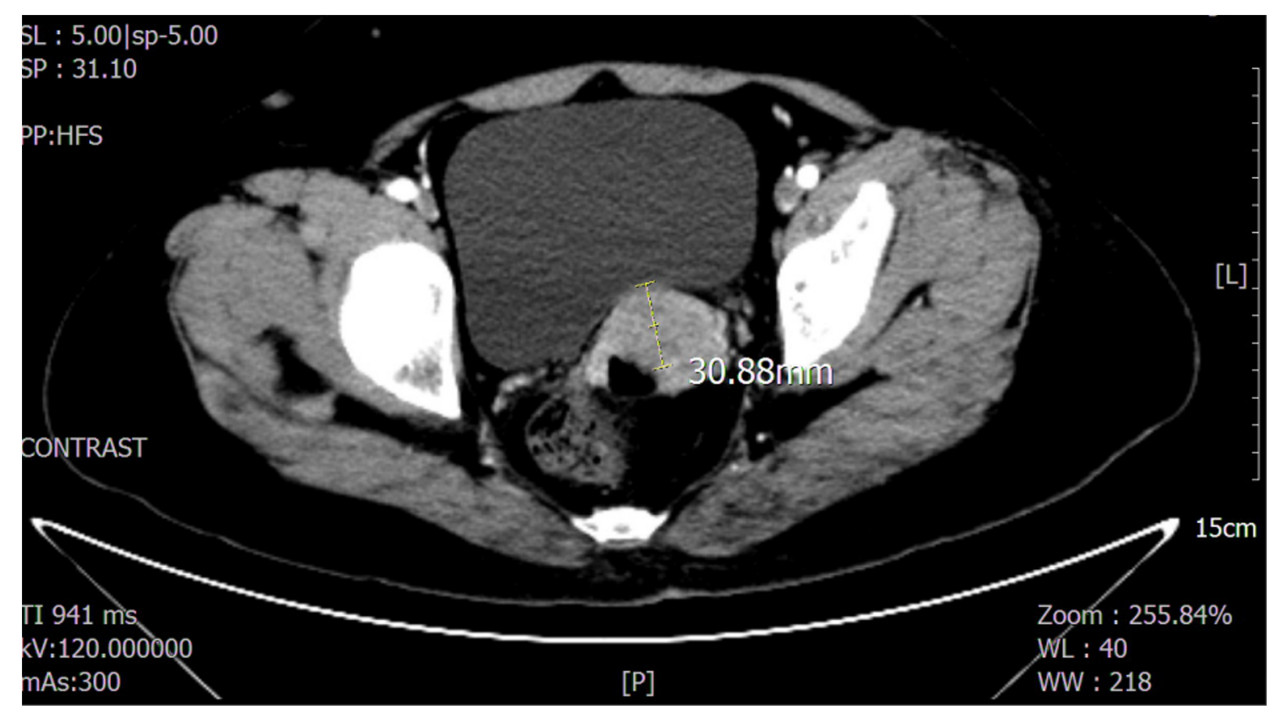

Figure I Pelvic CT scan showing asolid tumor with adiameter of $30.88 \mathrm{~mm}$ in the cervix. 

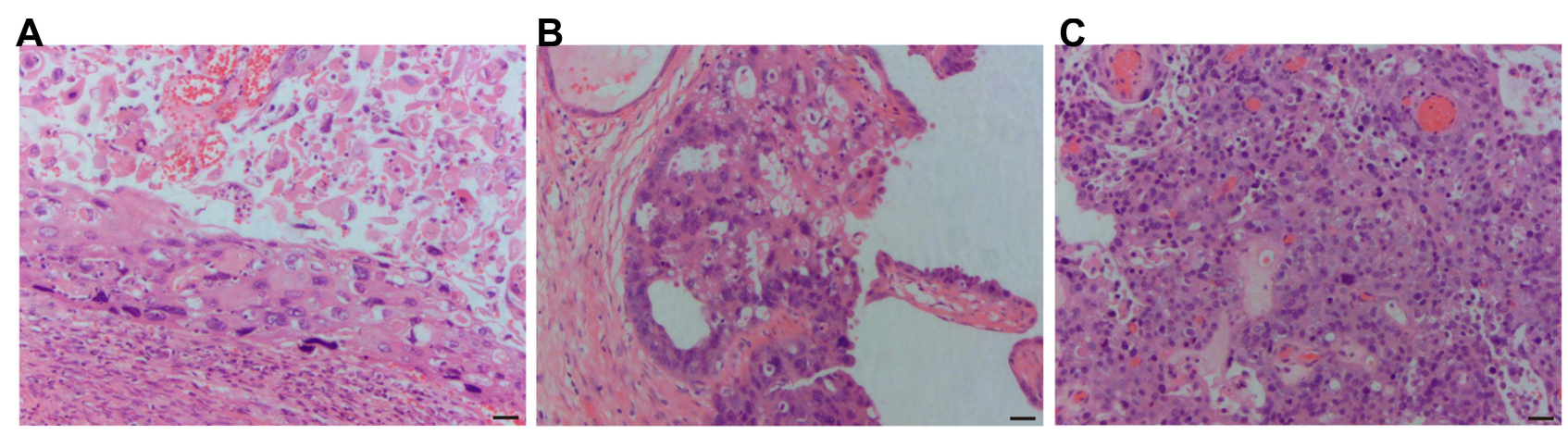

Figure 2 The H\&E staining of the cervical squamous cell carcinoma, right ovarian serous borderline tumors with intraductal carcinoma, right fallopian tube adenocarcinoma. (A) Cervical squamous cell carcinoma. The atypicality was obvious, the proportion of cytoplasm was increased, part of the nucleus was deeply stained, the chromatin was irregular, the nuclear membrane was gathered and the nuclear membrane was wrinkled (H\&E, $\times 100)$. (B) Right ovarian serous borderline tumors with intraductal carcinoma. The tumorous epithelial cells are arranged in layers to form acomplex branch of papillary and mesh-like structures, which protrude from the surface of the cystic cavity. The proliferating tumor cells form cell buds and fall off, and are free of the cystic cavity. The nucleus is heterotypic, and the cell nucleus is deeply stained with visible nucleoli $(\mathrm{H} \& E, \times 100)$. (C) Right fallopian tube adenocarcinoma. The cancer cells are flaky, complex and irregularly arranged, the papillary structure is reduced or disappeared, the cancer cells are closely arranged, the nuclear atypia is obvious, the staining is deep, the nuclear fission is increased and pathological nuclear division is seen (H\&E, $\times 100)$. Scale bar: $20 \mu \mathrm{m}$.

its intravenous use, the regimen was changed to five cycles of $210 \mathrm{mg}$ liposomal paclitaxel and $120 \mathrm{mg}$ nedaplatin. The patient is currently under treatment, showing good response.

In order to show the tumor information more clearly, the grades, stages and histopathological phenotypes of the four types tumor are listed in Table 1. The diagnosis and treatment for the patient has been organized as atimeline (Figure 3.)

Table I The Grades, Stages and Histopathological Phenotypes of the Four Types of Tumor

\begin{tabular}{|l|l|l|l|}
\hline $\begin{array}{l}\text { Tumor } \\
\text { Types }\end{array}$ & Grades & Stages & $\begin{array}{l}\text { Histopathological } \\
\text { Phenotypes }\end{array}$ \\
\hline $\begin{array}{l}\text { Breast } \\
\text { cancer }\end{array}$ & $\begin{array}{l}\text { IV (BI- } \\
\text { RADS) }\end{array}$ & TINOM0 & Invasive ductal carcinoma \\
\hline $\begin{array}{l}\text { Cervical } \\
\text { cancer }\end{array}$ & $\begin{array}{l}\text { T2aNOM0/ } \\
\text { lla2 (FIGO) }\end{array}$ & Squamous cell carcinoma \\
\hline $\begin{array}{l}\text { Ovarian } \\
\text { cancer }\end{array}$ & $I$ & $\begin{array}{l}\text { TIaNOM0/ } \\
\text { la (FIGO) }\end{array}$ & $\begin{array}{l}\text { Serous borderline tumor } \\
\text { and intraductal carcinoma }\end{array}$ \\
\hline $\begin{array}{l}\text { Fallopian } \\
\text { tube } \\
\text { cancer }\end{array}$ & $\begin{array}{l}\text { TIaNOM0/ } \\
\text { la (FIGO) }\end{array}$ & Adenocarcinoma \\
\hline
\end{tabular}

Abbreviations: BI-RADS, Breast Imaging-Reporting and Data System; FIGO, The International Federation of Gynecology and Obstetrics.

\section{Discussion}

The observation that synchronous MPC can occur in the same individual is of immense clinical interest. The definition of MPC varies. At present, the two commonly used definitions are provided by the Surveillance Epidemiology and End Results (SEER) project and the International Association of Cancer Registries and International Agency for Research on Cancer (IACR/IARC). ${ }^{3}$ Themain difference is the division of the tumor site area. For example, the colon is regarded as one site according to IACR/ IARC criteria, while the SEER considers single tumors of different parts of the colon as single tumors. ${ }^{1}$ Besides, the definition of synchronous and metachronous multiple primaries are also different. In the SEER criteria, a2-month period is recommended to distinguish between synchronous and metachronous multiple primaries, while an interval of 6 months is recommended in IARC rules. ${ }^{4}$ To distinguish between primary tumor and secondary tumor (like malignant tumors, recurrence, metastasis or local spread) in the same individual, the different histological presence of tumors is an important criterion., ${ }^{1,5,6}$ In our study, according to the above-mentioned criteria, the patient met the definition of MPC, namely the rare quadruple cancer, involving four sites of cancer (breast, cervix, ovary and fallopian tube).

Breast cancer ranks first among female malignant tumors in the Chinese population. According to data released by the National Cancer Center of China, there were approximately 278,900 new cases of female breast cancer in 2014, accounting for $16.51 \%$ of the incidence of female malignant tumors. ${ }^{7}$ Besides, the mortality rate 


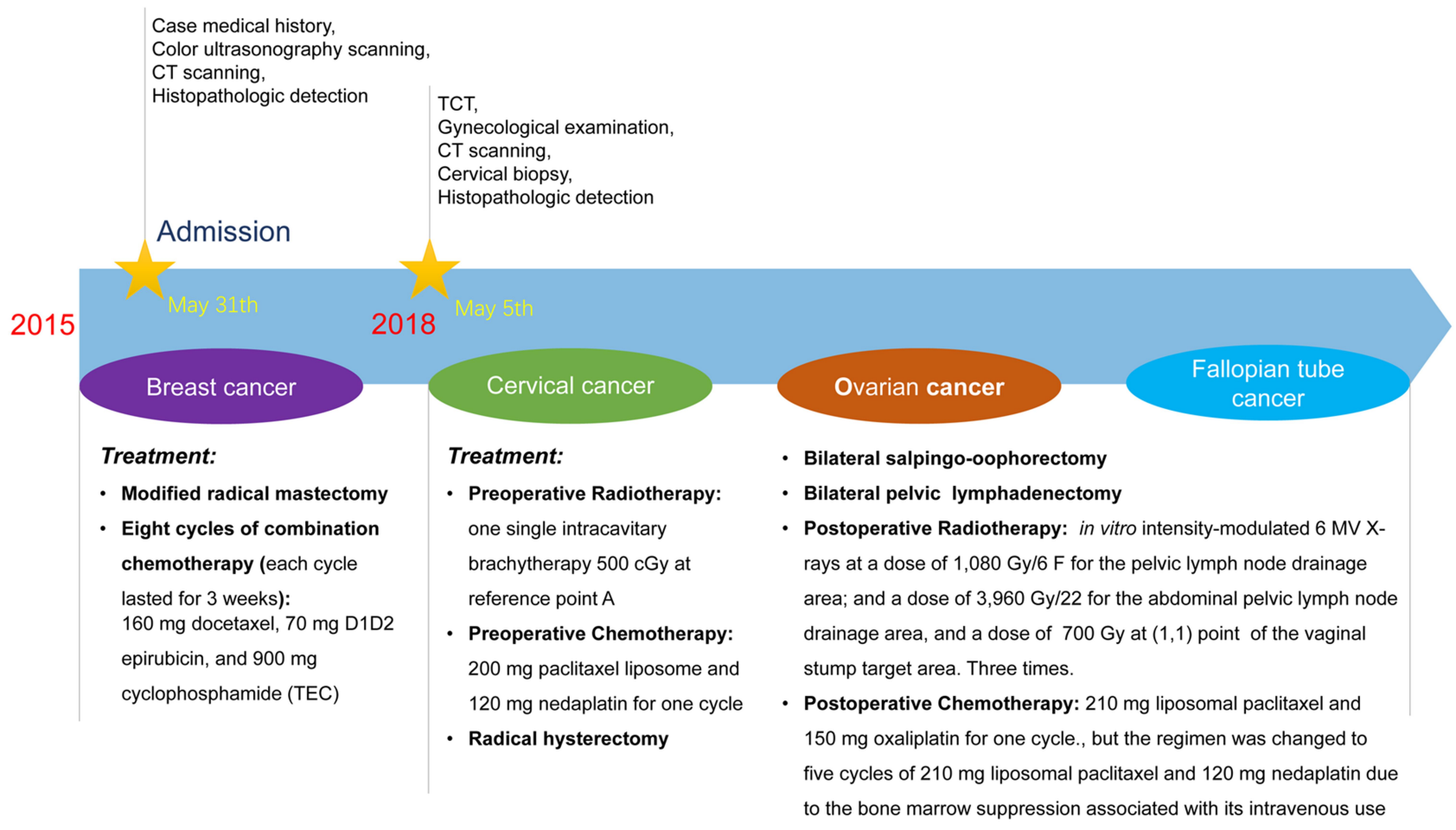

Figure 3 The timeline of diagnosis and treatment for the patient.

among older women is also high, and these patientstend to die from metastatic breast cancer or stage 4 breast cancer. ${ }^{8}$ Spread of breast cancer usually is to non-adjacent parts of the body, most commonly to the important sites, like bones, liver, lungs and/or brain. ${ }^{8}$ In one case report, they found that the pituitary gland, arare site, had become the metastasis organ of breast cancer via possibly hematogenous spread, which added to the difficulty of diagnosis, resulting in the delay of subsequent treatment. ${ }^{9}$ Hence, the identification of metastases is key to the treatment, and histology staining and immunohistochemistry are critical for the diagnosis. ${ }^{2}$ The earliest primary cancer of this patient was thebreast cancer, and the other primary cancers were located in the reproductive system. Therefore, the possibility of metastasis should be ruledout. According to the histopathological results, they belong to different carcinomasinvasive ductal carcinoma, squamous cell carcinoma, serous borderline tumor and intraductal carcinoma, adenocarcinoma, suggesting that these tumors had separate etiologies and were not metastatic tumors.

Aprevious study reported that endometrium and ovary are the common, among Japanese MPC patients with breast cancer, gallbladder and bile duct (12.8\%) and digestive tract ( $11.87 \%$ colorectal and $11.67 \%$ stomach) are the common site for the occurrence of another primary cancer while reproductive system were approximately $2-4.5 \%(2.05 \%$ uterine cervix, $3.99 \%$ ovary and $4.50 \%$ corpus uteri). ${ }^{10}$ Acase report similar to ours concerned a68-year-old female patient with quadruple primary cancer of the breast, rectum, ovary and endometrium. ${ }^{2}$ No previous studies have reported quadruple cancer consisting of breast cancer and three other concurrent types of female reproductive cancer: cervical, ovarian and fallopian tube cancers.

The etiology and pathogenesis of MPC remain unclear. In one published review article, the summary showed that genetic factors (eg BRCA1, BRCA2) are viewed as common risk factors for multiple primaries. ${ }^{1}$ In our case, the patient's family history suggested ahigh risk of malignancy. Her father died of liver cancer, and her aunt, who was still alive, was asurvivor of ovarian cancer. The patient was tested for tumor-specific druggable genes. There were 67 genes in the genetic test, 40 of which were recommended by NCCN guidelines, and the rest were selected based on their association with the risk of the corresponding genetic disease or recommended by the database. However, the results of the genetic test were all negative. It has been proposed that embryologically similar tissues may develop synchronous neoplasms in response to simultaneous exposure to hormones and carcinogens. ${ }^{11}$ Besides, malignancies arising in the same organ system often result from aprocess called "field cancerization", which is aconcept that explains 
multifocal growth of independent clones, probably as aresult of chronic exposure to environmental carcinogens. ${ }^{12}$ Although some literatures reported that therapy itself may be related to the occurrence of secondary cancers, ${ }^{13}$ the bigger possibility was an effect on the mobilization of the primary tumor. ${ }^{14}$ This patient was not exposed to any specific carcinogenic environmental factors after receiving chemotherapy for breast cancer 3 years ago, and she had anormal lifestyle. Previous literature has published that reproductive system infection and hormonal treatment might be prevalentMPC risk factors. ${ }^{15}$ Though the patient was positive for HPV-16, no endometriosis and endometrial carcinoma were found in this case, and there was not any hormonal treatment for the patient. Therefore, chemohormonal therapy or genetic predisposition probably did not induce quadruple cancer in our patient.

The patient developed breast cancer 3 years ago, and now she had triple genital cancer. Diagnosis and treatment of synchronous tumors are not different from those for single primary tumors. Curative resection is the effective approach in cases of MPC. ${ }^{16}$ Although prognosis may depend on the individual cancer, curative resection supposedly contributes to both an improved prognosis and better quality of life, and the therapy strategy is definitely individualized. ${ }^{15}$ In this case, the patient underwent accurate gene testing for targeted therapy. No relevant mutations were detected in genes related to drug resistance, immunosuppression sensitivity and immunotherapy super-progression. Moreover, the tumor mutational burden and tumor new antigen load of the patient were non-TMB-H and non-TNB-H, respectively, suggesting that the subject was less sensitive to immunosuppressants. Compared with metastatic lesions, most synchronous female malignancies are accompanied by early-stage and low-grade lesions with amore favorable prognosis. ${ }^{1}$

We concluded that our patient was at risk for recurrence or metastasis of each lesion as well as for the development of afifth cancer. This was an interesting case, and we will continue to follow up this patient. Our work might provide aseries of diagnostic, etiological and therapeutic strategies for MPC patients.

\section{Data Sharing Statement}

The data sets used and/or analyzed during this study can be obtained from the corresponding author Li Sun as reasonably required.

\section{Ethics Approval and Consent to Participate}

The study was approved by the Ethical Committees of Jinan Maternity and Child Care Hospital Affiliated to Shandong First Medical University. Written and signed informed consent was obtained from the patient to publish the case.

\section{Consent for Publication}

Not applicable.

\section{Acknowledgment}

Jing Bai and Zhaoguang Xie are co-first authors.

\section{Disclosure}

The authors declare that they have no competing interests.

\section{References}

1. VogtA, SchmidS, HeinimannK, etal. Multiple primary tumours: challenges and approaches, a review. ESMO Open. 2017;2(2):e000172. doi:10.1136/esmoopen-2017-000172

2. NohSK, YoonJY, RyooUN, etal. Acase report of quadruple cancer in asingle patient including the breast, rectum, ovary, and endometrium. JGynecol Oncol. 2008;19(4):265-269.

3. CoyteA, MorrisonDS, McLooneP. Second primary cancer risk - the impact of applying different definitions of multiple primaries: results from aretrospective population-based cancer registry study. $B M C$ Cancer. 2014;14:272. doi:10.1186/1471-2407-14-272

4. AmerMH. Multiple neoplasms, single primaries, and patient survival. Cancer Manag Res. 2014;6:119-134. doi:10.2147/CMAR.S57378

5. ZhaiC, CaiY, LouF, etal. Multiple primary malignant tumors - aclinical analysis of 15,321 patients with malignancies at asingle center in China. JCancer. 2018;9(16):2795-2801. doi:10.7150/jca.25482

6. BanimostafaviES, TayebiS, TayebiM, MontazerF. Case report: synchronous primary malignancy including the breast and endometrium. F1000Research. 2017;6:1502. doi:10.12688/ f1000research.11971.1

7. ChenW Current situation and trend of malignant tumor in China. Paper presented at: the 9th Chinese Congress of internal medicine of oncology, the 4th China oncologist conference, the 2015 annual meeting of cancer clinical chemotherapy Committe of China Anti Cancer Association 2015; Beijing City, China.

8. CardosoF, SpenceD, MertzS, etal. Global analysis of advanced/metastatic breast cancer: decade report (2005-2015). Breast. 2018;39:131-138. doi:10.1016/j.breast.2018.03.002

9. SpinelliG, Lo RussoG, MieleE, etal. Breast cancer metastatic to the pituitary gland: acase report. World JSurgical Oncology. 2012;10:137. doi:10.1186/1477-7819-10-137

10. MikiY, SugawaraY, ShibaharaY, TsujiI, SasanoH, ItoK. Multiple primary cancers associated with endometrial and ovarian cancers: an analysis based upon the japan autopsy annual database from 2002 to 2010. JObstet Gynaecol Res. 2019;45(5):1012-1018. doi:10.1111/ jog. 13934

11. AydinerA, KaradenizA, UygunK, etal. Multiple primary neoplasms at asingle institution: differences between synchronous and metachronous neoplasms. Am JClinical Oncology. 2000;23(4):364-370. doi:10.1097/00000421-200008000-00011 
12. CurtiusK, WrightNA, GrahamTA. An evolutionary perspective on field cancerization. Nature Reviews Cancer. 2018;18(1):19-32.

13. CoM, LeeA, KwongA. Cutaneous angiosarcoma secondary to lymphoedema or radiation therapy - asystematic review. Clinical Oncol. 2019;31(4):225-231. doi:10.1016/j.clon.2019.01.009

14. MartinOA, AndersonRL, NarayanK, MacManusMP. Does the mobilization of circulating tumour cells during cancer therapy cause metastasis? Nature Reviews Clinical Oncology. 2017;14(1):32-44.

15. CopurMS, ManapuramS. Multiple primary tumors over a lifetime. Oncology. 2019;33:7.
16. ZainoR, WhitneyC, BradyMF, DeGeestK, BurgerRA, BullerRE. Simultaneously detected endometrial and ovarian carcinomas-a prospective clinicopathologic study of 74 cases: agynecologic oncology group study. Gynecol Oncol. 2001;83(2):355-362. doi:10.1006/ gyno.2001.6400

\section{Publish your work in this journal}

The International Journal of General Medicine is an international, peer-reviewed open-access journal that focuses on general and internal medicine, pathogenesis, epidemiology, diagnosis, monitoring and treatment protocols. The journal is characterized by the rapid reporting of reviews, original research and clinical studies across all disease areas. The manuscript management system is completely online and includes a very quick and fair peer-review system, which is all easy to use. Visit http://www.dovepress.com/ testimonials.php to read real quotes from published authors. 